Proceedings of the Edinburgh Mathematical Society (2003) 46, 679-686 (C)

DOI:10.1017/S0013091501000839 Printed in the United Kingdom

\title{
ON $M$-STRUCTURE AND WEAKLY COMPACTLY GENERATED BANACH SPACES
}

\author{
EDUARDO NIETO \\ Departamento de Análisis Matemático, Facultad de Ciencias, \\ Universidad de Granada, 18071 Granada, \\ Spain (enieto@ugr.es)
}

(Received 11 September 2001)

\begin{abstract}
It is well known that every non-reflexive $M$-ideal is weakly compactly generated (in short, WCG). We present a family of Banach spaces $\left\{V_{s}: 0<s<1\right\}$ which are not WCG and such that every $V_{s}$ satisfies the inequality

$$
\|\varphi\| \geqslant\|\pi \varphi\|+s\|\varphi-\pi \varphi\| \quad \forall \varphi \in V_{s}^{* * *},
$$

where $\pi$ is the canonical projection from $V_{s}^{* * *}$ onto $V_{s}^{*}$. In particular, no $V_{s}$ can be renormed to be an
\end{abstract} $M$-ideal.

Keywords: $M$-ideal; $M(r, s)$-inequality; weakly compactly generated (WCG) Banach space

2000 Mathematics subject classification: Primary 46B20

\section{Introduction}

Let $X$ be a Banach space. Recall that $X$ is an $M$-ideal if

$$
\|\varphi\|=\|\pi \varphi\|+\|\varphi-\pi \varphi\| \quad \forall \varphi \in X^{* * *}
$$

where $\pi$ denotes the canonical projection from $X^{* * *}$ onto $X^{*}$ (for complete information about $M$-ideals, see [6]). $X$ is said to be weakly compactly generated (in short, WCG) if $X$ is the closed linear span of a weakly compact subset of $X$. Fabian and Godefroy showed in [4] (cf. [6]) that every non-reflexive $M$-ideal is WCG. Indeed, it is proved in $[\mathbf{1}]$ that if $X$ is an Asplund space with the property that for each $\varphi \in X^{* * *},\|\varphi-\pi \varphi\|<\|\varphi\|$ whenever $\pi \varphi \neq 0$, then $X$ is WCG.

Following [1] and [2], a Banach space $X$ satisfies the $M(r, s)$-inequality if

$$
\|\varphi\| \geqslant r\|\pi \varphi\|+s\|\varphi-\pi \varphi\| \quad \forall \varphi \in X^{* * *}
$$

holds for given $r, s \in] 0,1]$. It is clear that the notion of $M(1,1)$-inequality coincides with that of $M$-ideal.

Following Johnson and Lindenstrauss [7], for every $s \in] 0,1[$, we present a Banach space $V_{s}$ which is not WCG and which satisfies the $M(1, s)$-inequality, resolving the open 
question formulated in [1] of whether there are Banach spaces satisfying the $M(1, s)$ inequality without being WCG. As a consequence, $V_{s}$ cannot be renormed to be an $M$ ideal (see Remarks 2.6 below). In Remarks 2.6, we collect some properties derived from the $M(1, s)$-inequality. For instance, we show that for every $s \in] 0,1\left[, V_{s}^{*}\right.$ contains no proper norming subspaces, $V_{s}$ is an Asplund space, $V_{s}$ has the unique extension property, $V_{s}^{*}=\overline{\operatorname{lin}}\left(w^{*}-\operatorname{sexp} B_{V_{s}^{*}}\right)$ (where $w^{*}$-sexp $B_{V_{s}^{*}}$ denotes the set of the weak* strongly exposed points of $B_{V_{s}^{*}}$ ), and $V_{s}$ has property $(u)$ of Pełczyński.

In a Banach space $X$, the closed unit ball is denoted by $B_{X}$. The Banach space of all bounded linear operators on $X$ will be denoted by $\mathcal{L}(X)$. For a set $A \subset X$, we denote by $\operatorname{lin} A$ its linear span.

In the next section, we exhibit some techniques for constructing Banach spaces satisfying the $M(r, s)$-inequality.

\section{Example}

First, we show some results which will be a key tool in the construction of examples of Banach spaces satisfying the $M(r, s)$-inequality. The next result generalizes a result in $[8]$.

Proposition 2.1. Let $X$ be a Banach space and $r, s \in] 0,1]$. If there exists a net $\left(V_{\alpha}\right)$ in $\mathcal{L}\left(X^{* *}\right)$ satisfying

(1) for each $\alpha, \operatorname{Im} V_{\alpha} \subset X$;

(2) for each $x^{*} \in X^{*}$ and $x^{* *} \in X^{* *}, \lim _{\alpha} x^{*}\left(V_{\alpha} x^{* *}\right)=x^{* *} x^{*}$; and

(3) for each $x \in B_{X}$ and $x^{* *} \in B_{X^{* *}}$,

$$
\limsup \left\|r V_{\alpha} x+s\left(x^{* *}-V_{\alpha} x^{* *}\right)\right\| \leqslant 1
$$

then $X$ satisfies the $M(r, s)$-inequality.

Proof. Let $\varepsilon>0$ and $\varphi \in X^{* * *}$, and consider $x^{* *} \in B_{X^{* *}}$ and $x \in B_{X}$ such that

$$
\pi \varphi(x)>\|\pi \varphi\|-\varepsilon, \quad(\varphi-\pi \varphi)\left(x^{* *}\right)>\|\varphi-\pi \varphi\|-\varepsilon .
$$

By (2) and (3), there is $\alpha$ sufficiently large such that

$$
\begin{aligned}
\pi \varphi\left(V_{\alpha} x\right) & >\|\pi \varphi\|-\varepsilon, \\
\left\|r V_{\alpha} x+s\left(x^{* *}-V_{\alpha} x^{* *}\right)\right\| & \leqslant 1+\varepsilon \\
\left|\pi \varphi\left(x^{* *}-V_{\alpha} x^{* *}\right)\right| & <\varepsilon
\end{aligned}
$$

Hence,

$$
\begin{aligned}
(1+\varepsilon)\|\varphi\| & \geqslant \varphi\left(r V_{\alpha} x+s\left(x^{* *}-V_{\alpha} x^{* *}\right)\right) \\
& =r \pi \varphi\left(V_{\alpha} x\right)+s\left(\pi \varphi\left(x^{* *}-V_{\alpha} x^{* *}\right)+(\varphi-\pi \varphi)\left(x^{* *}-V_{\alpha} x^{* *}\right)\right) \\
& >r(\|\pi \varphi\|-\varepsilon)-\varepsilon s+s(\|\varphi-\pi \varphi\|-\varepsilon) .
\end{aligned}
$$

Letting $\varepsilon \rightarrow 0$, the result follows. 
Corollary 2.2. Let $X$ be a Banach space and $r, s \in] 0,1]$. If there exists a net $\left(T_{\alpha}\right)$ in $\mathcal{L}(X)$ satisfying

(1) for each $\alpha, \operatorname{Im} T_{\alpha}^{* *} \subset X$;

(2) for each $x^{*} \in X^{*}$ and $x^{* *} \in X^{* *}, \lim _{\alpha} x^{*}\left(T_{\alpha}^{* *} x^{* *}\right)=x^{* *} x^{*}$; and

(3) for each $x \in B_{X}$,

$$
\limsup _{\alpha} \sup _{y \in B_{X}}\left\|r T_{\alpha} x+s\left(y-T_{\alpha} y\right)\right\| \leqslant 1
$$

then $X$ satisfies the $M(r, s)$-inequality.

Proof. This follows from the weak* density of $B_{X}$ in $B_{X^{* *}}$ and the above result.

Proposition 2.3. Let $X$ be a Banach space, $r, s \in] 0,1]$ and a net $\left(T_{\alpha}\right)$ in $\mathcal{L}(X)$. The following assertions are equivalent.

(1) $\limsup \sup _{\alpha, y \in B_{X}}\left\|r T_{\alpha} x+s\left(y-T_{\alpha} y\right)\right\| \leqslant 1$.

(2) $\limsup \sup _{\alpha}\left(r\left\|T_{\alpha}^{*} x^{*}\right\|+s\left\|B_{X^{*}}^{*}-T_{\alpha}^{*} x^{*}\right\|\right) \leqslant 1$.

Proof. For each $\alpha$, consider the operator $V_{\alpha}: X \oplus_{\infty} X \longrightarrow X$ defined by

$$
V_{\alpha}(x, y)=r T_{\alpha} x+s\left(y-T_{\alpha} y\right) \quad \forall x, y \in X .
$$

Then (1) means that $\lim \sup _{\alpha}\left\|V_{\alpha}\right\| \leqslant 1$, and (2) means that $\lim \sup _{\alpha}\left\|V_{\alpha}^{*}\right\| \leqslant 1$.

Now we present a Banach space $V$ similar to the Banach space $U$ given in [7, Example 1]. Let $\left\{N_{\gamma}: \gamma \in \Gamma\right\}$ be a collection of infinite subsets of the integers such that $N_{\gamma} \cap N_{\gamma^{\prime}}$ is finite for $\gamma \neq \gamma^{\prime}$, and such that $\Gamma$ has the cardinality of the continuum. Denote

$$
V_{0}=\left\{(y, z) \in \ell_{\infty} \bigoplus_{\infty} c_{00}(\Gamma): \lim _{n}\left(y(n)-\sum_{\gamma \in \Gamma_{n}} z(\gamma)\right)=0\right\},
$$

where for each $n \in \mathbb{N}, \Gamma_{n}=\left\{\gamma \in \Gamma: n \in N_{\gamma}\right\}, \ell_{\infty}$ and $c_{00}(\Gamma)$ are equipped with the usual sup norm $\|\cdot\|_{\infty} . V$ denotes the completion of $V_{0}$.

Observe that, given $(y, z) \in V_{0}$, we have that for each $\gamma \in \Gamma, z(\gamma)=\lim _{n \in N_{\gamma}} y(n)$. So, $\|(y, z)\|=\|y\|_{\infty}$. On the other hand, note that the functionals $(y, z) \mapsto y(n), n \in \mathbb{N}$, form a total set in $V_{0}^{*}$. Hence, $V^{*}$ is $w^{*}$-separable. Therefore, the proof of the next result follows from the one given in [7, Example 1].

Proposition 2.4. $V$ satisfies the following properties.

(1) $V$ has a subspace $X$ isometric to $c_{0}$ such that $V / X$ is isometric to $c_{0}(\Gamma)$.

(2) $V$ is not WCG.

(3) $V^{*}$ is isomorphic to $\ell_{1} \oplus \ell_{1}(\Gamma)$ and thus is not $W C G$. 
Theorem 2.5. For every $s \in] 0,1\left[, V\right.$ admits an equivalent norm $\|\cdot\|_{s}$ for which $V_{s}=\left(V,\|\cdot\|_{s}\right)$ satisfies the $M(1, s)$-inequality.

Proof. Let $s \in] 0,1[$, and define

$$
a_{s}=\left\{\begin{array}{ll}
(1-s) /(1+s) & \text { if } s \leqslant \frac{1}{2}, \\
(1-s)\left(1+2 s^{2}\right) & \text { if } s>\frac{1}{2},
\end{array} \quad \text { and } \quad b_{s}=s a_{s} .\right.
$$

It is straightforward to show that

$$
a_{s}+s\left(1+\max \left\{2 b_{s}, a_{s}\right\}\right)=1 .
$$

Now consider in $\ell_{1} \oplus \ell_{1}(\Gamma)$ the following norm:

$$
\left\|\left(y^{*}, z^{*}\right)\right\|_{s}=\left\|y^{*}\right\|_{\ell_{1}}+\max \left\{b_{s}\left(\left\|y^{*}\right\|_{\ell_{1}}+\left\|z^{*}\right\|_{\ell_{1}(\Gamma)}\right), a_{s}\left\|z^{*}\right\|_{\ell_{1}(\Gamma)}\right\},
$$

for all $y^{*} \in \ell_{1}, z^{*} \in \ell_{1}(\Gamma)$.

Following the correction given by Johnson and Lindenstrauss in [7], it is easy to show that $\|\cdot\|_{s}$ is an equivalent dual norm on $V^{*}$. In fact, from the form of the duality between $V$ and $V^{*}$, it follows that the norm $\|\cdot\|_{s}$ is weak*-lower semicontinuous. As a consequence, $V$ has an equivalent norm whose dual norm on $V^{*}$ coincides with $\|\cdot\|_{s}$ (see $[\mathbf{3}$, Theorem 3 , p. 106]).

We will now prove that $V_{s}$ satisfies the $M(1, s)$-inequality. In fact, consider the set

$$
A=\{(n, F): n \in \mathbb{N}, F \subset \Gamma, F \text { finite }\},
$$

ordered as follows: $(n, F) \leqslant(m, G)$ if $n \leqslant m$ and $F \subset G$. For each $F \subset \Gamma$, we define

$$
N_{F}=\bigcup_{\gamma \in F} N_{\gamma},
$$

and for each $(n, F) \in A$, we consider $P_{n, F}: V \longrightarrow V$ defined as follows: for $(y, z) \in V_{0}$, $P_{n, F}(y, z)=\left(y_{0}, z_{0}\right)$, where

$$
y_{0}(m)= \begin{cases}y(m) & \text { if } m \leqslant n, \\ \sum_{\gamma \in \Gamma_{m} \cap F} z(\gamma) & \text { if } m>n \text { and } m \in N_{F}, \\ 0 & \text { otherwise }\end{cases}
$$

and

$$
z_{0}(\gamma)= \begin{cases}z(\gamma) & \text { if } \gamma \in F \\ 0 & \text { if } \gamma \notin F\end{cases}
$$

We extend $P_{n, F}$ to $V$ by continuity. 
We prove that for each $(n, F), \operatorname{Im} P_{n, F}^{* *} \subset V$. In fact, it is clear that if $(y, z) \in V^{* *}=$ $\ell_{\infty} \oplus \ell_{\infty}(\Gamma)$, then $P_{n, F}^{* *}(y, z)=\left(y_{0}, z_{0}\right)$, where

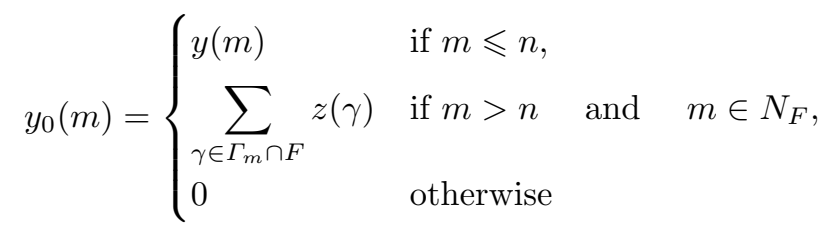

and

$$
z_{0}(\gamma)= \begin{cases}z(\gamma) & \text { if } \gamma \in F \\ 0 & \text { if } \gamma \notin F\end{cases}
$$

So, $P_{n, F}^{* *}(y, z) \in V$.

We now show that for each $x \in V$ and $\left(y^{*}, z^{*}\right) \in V^{*},\left\|x-P_{n, F}(x)\right\| \rightarrow 0$ and $\|\left(y^{*}, z^{*}\right)-$ $P_{n, F}^{*}\left(y^{*}, z^{*}\right) \|_{s} \rightarrow 0$. Indeed, let $\varepsilon>0$, and suppose that $x=(y, z) \in V_{0}$. For $(n, F)$ large enough, we have that $\{\gamma \in \Gamma: z(\gamma) \neq 0\} \subset F$ and

$$
\begin{array}{r}
\left|y(n)-\sum_{\gamma \in \Gamma_{n} \cap F} z(\gamma)\right|<\varepsilon \quad \text { if } n \in N_{F}, \\
|y(n)|<\varepsilon \quad \text { if } n \notin N_{F} .
\end{array}
$$

So, $\left\|(y, z)-P_{n, F}(y, z)\right\|<\varepsilon$.

On the other hand, it is easy to show that $P_{n, F}^{*}\left(y^{*}, z^{*}\right)=\left(y_{0}^{*}, z_{0}^{*}\right)$, where

$$
y_{0}^{*}(m)= \begin{cases}y^{*}(m) & \text { if } m \leqslant n, \\ 0 & \text { if } m>n\end{cases}
$$

and

$$
z_{0}^{*}(\gamma)= \begin{cases}\sum_{m>n, m \in N_{\gamma}} y^{*}(m)+z^{*}(\gamma) & \text { if } \gamma \in F, \\ 0 & \text { if } \gamma \notin F .\end{cases}
$$

Hence,

$$
\begin{aligned}
& \left\|\left(y^{*}, z^{*}\right)-P_{n, F}^{*}\left(y^{*}, z^{*}\right)\right\|_{s} \\
& \leqslant \sum_{m=n+1}^{\infty}\left|y^{*}(m)\right| \\
& \quad+\max \left\{b_{s}\left(2 \sum_{m=n+1}^{+\infty}\left|y^{*}(m)\right|+\sum_{\gamma \notin F}\left|z^{*}(\gamma)\right|\right), a_{s}\left(\sum_{m=n+1}^{+\infty}\left|y^{*}(m)\right|+\sum_{\gamma \notin F}\left|z^{*}(\gamma)\right|\right)\right\} .
\end{aligned}
$$

We obtain the desired conclusion by taking $(n, F)$ sufficiently large that

$$
\sum_{m=n+1}^{+\infty}\left|y^{*}(m)\right|<\varepsilon \quad \text { and } \quad \sum_{\gamma \notin F}\left|z^{*}(\gamma)\right|<\varepsilon .
$$


Finally, we prove that $\left(P_{n, F}\right)$ satisfies condition (2) of Proposition 2.3 for $r=1$ and $s$. In fact, by $(*)$, for each $(n, F) \in A$ and $\left(y^{*}, z^{*}\right) \in V^{*}$, we have that

$$
\begin{aligned}
& \left\|P_{n, F}^{*}\left(y^{*}, z^{*}\right)\right\|_{s}+s\left\|\left(y^{*}, z^{*}\right)-P_{n, F}^{*}\left(y^{*}, z^{*}\right)\right\|_{s} \\
& \leqslant \sum_{m=1}^{n}\left|y^{*}(m)\right| \\
& \quad+\max \left\{b_{s}\left(\sum_{m=1}^{n}\left|y^{*}(m)\right|+\sum_{m=n+1}^{+\infty}\left|y^{*}(m)\right|+\sum_{\gamma \in F}\left|z^{*}(\gamma)\right|\right),\right. \\
& \quad+s \sum_{m=n+1}^{+\infty}\left|y^{*}(m)\right| \\
& \quad+s \max \left\{b_{s}\left(2 \sum_{m=n+1}^{+\infty}\left|y^{*}(m)\right|+\sum_{\gamma \in F}\left|z^{*}(\gamma)\right|\right)\right\} \\
& \leqslant \sum_{n=1}^{+\infty}\left|y^{*}(m)\right|+\left(a_{s}+s\left(1+\max \left\{2 b_{s}, a_{s}\right\}\right)\right) \sum_{m=n+1}^{+\infty}\left|y^{*}(m)\right| \\
& \left.\left.\quad+\sum_{m \notin F}\left|z^{*}(\gamma)\right|\right), a_{s}\left(\sum_{m=n+1}^{+\infty}\left|y^{*}(m)\right|+\sum_{\gamma \notin F}\left|z^{*}(\gamma)\right|\right)\right\} \\
& \leqslant\left\|y^{*}\right\|_{\ell_{1}}+\max \left\{b_{s}\left(\sum_{m=1}^{n}\left|y^{*}(m)\right|+\sum_{\gamma \in \Gamma}\left|z^{*}(\gamma)\right|\right), a_{s} \sum_{\gamma \in \Gamma}\left|z^{*}(\gamma)\right|\right\}
\end{aligned}
$$

\section{Remarks 2.6.}

(1) Since every non-reflexive $M$-ideal is WCG [4, Theorem 3] (cf. [6, Theorem 4.6, p. 142]), by Proposition $2.4, V_{s}$ cannot be renormed to be an $M$-ideal.

(2) Note that $\|\cdot\|_{s}$ on $V$ is not Fréchet differentiable. In fact, this follows from the equality

$$
\left(1+b_{s}\right)\|x\|_{s}=\|x\|_{\infty} \quad \forall x \in c_{0} .
$$

(3) Finally, by the $M(1, s)$-inequality, some properties of $V_{s}$ are immediate. In fact, by $[\mathbf{1}$, Proposition 2.5] and [5, Proposition 2.5], we have

(i) $V_{s}^{*}$ contains no proper norming subspaces and $V_{s}$ is an Asplund space;

(ii) $V_{s}$ has the unique extension property;

(iii) if $X$ is a closed subspace of $V_{s}$ such that there exists a Banach space $Y$ with Banach-Mazur distance $d\left(X, Y^{*}\right)<1+s / 2$, then $X$ is reflexive. 
By [1, Corollary 2.8], we obtain

(i) $V_{s}$ does not contain an isomorphic copy of $\ell_{1}$;

(ii) if $X$ is a Banach space such that $V_{s} \subsetneq X \subset V_{s}^{* *}$, then there are no norm-one projections from $X$ onto $V_{s}$;

(iii) every subspace or quotient of $V_{s}$ which is isometric to a dual space is reflexive.

By [1, Theorem 3.1], we have

(i) $V_{s}$ has property $U$ of Phelps;

(ii) $V_{s}$ has property $(u)$ of Pełczyński with constant $k_{u}\left(V_{s}\right) \leqslant 1 / s$.

By [1, Corollary 3.4], the following results hold.

(i) Every subspace of $V_{s}$ has property $(V)$ of Pełczyński. In particular, $V_{s}$ is not weakly sequentially complete and $V_{s}$ fails the Radon-Nikodým property.

(ii) $V_{s}^{*}$ is weakly sequentially complete. (In fact, since $V_{s}^{*}$ is isomorphic to $\ell_{1} \oplus$ $\ell_{1}(\Gamma), V_{s}^{*}$ has the Schur property.)

(iii) $V_{s}$ is not complemented in $V_{s}^{* *}$.

(iv) $V_{s}^{* *} / V_{s}$ is not separable.

(v) Every operator from $V_{s}$ to a space not containing $c_{0}$ (in particular, every operator from $V_{s}$ to $V_{s}^{*}$ ) is weakly compact.

By $\left[\mathbf{1}\right.$, Theorem 3.6], we have that every slice of $B_{V_{s}}$ has diameter greater than or equal to $2 s$. In particular, $B_{V_{s}}$ is not dentable.

By [2, Proposition 2.1], we have

$$
V_{s}^{*}=\varlimsup \operatorname{lin}\left(w^{*}-\operatorname{sexp} B_{V_{s}^{*}}\right)
$$

Acknowledgements. The author is greatly indebted to M. González for suggesting the example. This work was supported by Junta de Andalucia grant FQM290.

\section{References}

1. J. C. Cabello and E. Nieto, On Properties of M-ideals, Rocky Mt. J. Math. 28 (1998), 61-93.

2. J. C. Cabello, E. Nieto And E. OJA, On ideals of compact operators satisfying the M(r,s)-inequality, J. Math. Analysis Applic. 220 (1998), 334-348.

3. J. Diestel, Geometry of Banach spaces - selected topics, Lecture Notes in Mathematics, vol. 485 (Springer, 1975).

4. M. Fabian And G. Godefroy, The dual of every Asplund space admits a projectional resolution of the identity, Studia Math. 91 (1988), 141-151.

5. G. Godefroy AND D. SAPhar, Duality in spaces of operators and smooth norms in Banach spaces, Illinois J. Math. 32 (1988), 672-695.

6. P. Harmand, D. Werner And W. Werner, M-ideals in Banach spaces and Banach algebras, Lecture Notes in Mathematics, vol. 1547 (Springer, 1993). 
7. W. B. Johnson AND J. Lindenstrauss, Some remarks on weakly compactly generated Banach space, Isr. J. Math. 17 (1974), 219-230 (Corrigendum: Isr. J. Math. 32 (1979), 382-383).

8. E. OJA, On the uniqueness of the norm-preserving extension of a linear functional in the Hahn-Banach theorem, Izv. Akad. Nauk Est. SSSR 33 (1984), 424-438 (in Russian). 